\title{
MicroRNA-21 regulates the migration and invasion of a stem-like population in hepatocellular carcinoma
}

\author{
LIANG ZHOU ${ }^{1 *}$, ZHAO-XU YANG ${ }^{2 *}$, WEN-JIE SONG ${ }^{2 *}$, QING-JUN LI ${ }^{2}$, \\ FAN YANG $^{2}$, DE-SHENG WANG ${ }^{2}$, NING ZHANG ${ }^{2}$ and KE-FENG DOU ${ }^{2}$ \\ ${ }^{1}$ Department of General Surgery, The 155 Central Hospital of PLA, Kaifeng, \\ He'nan 471000; ${ }^{2}$ Department of Hepatobiliary Surgery, Xijing Hospital, \\ The Fourth Military Medical University, Xi'an, Shannxi 710032, P.R. China
}

Received December 20, 2012; Accepted March 1, 2013

DOI: 10.3892/ijo.2013.1965

\begin{abstract}
Due to invasion and intrahepatic metastasis, the prognosis for patients with hepatocellular carcinoma (HCC) is poor. However, the mechanisms underlying these processes of HCC remain unclear. Cancer stem cells may be involved in early systemic dissemination and metastasis formation and side population (SP) cells isolated from diverse cancer cells possess stem cell-like properties. However, the mechanisms involved in migration and invasion of cancer stem cells are not well understood. In this study, we identified and isolated populations of SP cells from HCC cell lines using flow cytometry. SP cells showed higher levels of migration and invasion capability. Higher expression of miR-21 was observed in SP cells. Silencing of miR-21 led to a reduction in the migration and invasion of these cells and overexpression of miR-21 can increase in cell migration and invasion. Overexpression of miR-21 did not cause degradation of PTEN or RECK or PDCD4 mRNA but drastically inhibited its protein expression. Consistent with these results, silencing miR-21 increased the levels of PTEN, RECK and PDCD4 protein, respectively. The role of silencing miR-21 was partially attenuated by silencing of PTEN or RECK or PDCD4 mRNA. The results of this study revealed the aberrant expression of miR-21 in SP cells and showed that miR-21 regulates the expression of multiple target proteins that are associated with tumor dissemination. MiR-21 is a pro-metastatic miRNA in SP cells and raises the possibility that therapy of HCC may be improved by pharmaceutical strategies directed towards miR-21.
\end{abstract}

Correspondence to: Professor Ke-Feng Dou or Dr Ning Zhang, Department of Hepatobiliary Surgery, Xijing Hospital, The Fourth Military Medical University, Changlexi Road, Xi'an, Shannxi 710032, P.R. China

E-mail: kefengdou@126.com

E-mail: ningzhang912@163.com

*Contributed equally

Key words: hepatocellular carcinoma, microRNA-21, cancer stem cell, side population, migration, invasion

\section{Introduction}

Hepatocellular carcinoma (HCC) is one of the most common malignancies. Despite advances in the detection and treatment of $\mathrm{HCC}$, the mortality rate remains high because the majority of HCC patients with local invasion and intrahepatic metastasis for which most potentially curative therapies have limited efficacy (1). However, it may be an important therapeutic target for inhibition of invasion and metastasis in HCC.

During recent years, various studies have described cancer stem cell (CSC) hypothesis and these cells have heterogeneous tumorigenic potential. Based on this hypothesis, only a fraction of cells within a tumor is endowed with stem cell-like features, including unlimited proliferative potential and asymmetric cell division. This cellular subset, varying in size in different tumors, can initiate and sustain tumor growth and is believed to drive relapse (2). According to the CSC hypothesis solely the CSC population is responsible for early systemic dissemination and metastasis formation (3). So it is important to explore the potential mechanism of invasion and metastasis in CSC for therapy of HCC. Prior reports have suggested that side population (SP) cells separated from diverse cancer cells possess stem cell-like properties (4-6). SP cells refer to a unique cell population, first identified in bone marrow cells by Goodell et al, which could efflux the DNA-binding dye Hoechst 33342 (7). Thereafter, SP cells have been defined in multiple species and tissues and SP cells could be characterized as CSC in primary tissues of cancer. SP cells were also shown in established tumor cell lines with different origins, such as glioma (8), breast (9) and thyroid cancer monoclonal cell lines (10). In a previous study, we reported the identification and isolation of SP cell phenotype in human HCC cell lines that demonstrate specific characteristics of CSC when compared with MP cells, such as quiescence, elevated chemoresistance, increased tumorigenicity, higher actin polymerization ability and increased migration capacity towards the chemokine CXCL12 (11). However, it is unknown why SP cells have more migration and invasion capabilities.

A recently identified class of non-coding small RNAs, microRNAs (miRNAs), is a group of RNAs of 14-24 nucleotides that can negatively regulate protein expression at the posttranscriptional level by translational inhibition and/or mRNA 
degradation (12). Dysregulated expression of microRNAs has been identified in a variety of human malignancies and is suggested to have a significant role in the development or progression of tumors by inhibiting tumor suppressor genes or activating oncogenes. Among them, microRNA-21 (miR-21) has been widely suggested to be oncogenic in many tumors. It had demonstrated that miR-21 can increase tumor cells migration and invasion by directly targeting PTEN, RECK and PDCD4 (13-16). Although miR-21 and its target have been widely explored as a cancer-related target for tumors including $\mathrm{HCC}$, there is no information available concerning the relevance of the genes for metastasis in CSC. The aims of this study were to examine miR-21 in SP cells of HCC and to investigate the potential mechanism that miR-21 participated in the migration and invasion of SP cells.

\section{Materials and methods}

Cell culture and reagents. The human liver non-tumor cell line (HL-7702) and human HCC cell lines (SMMC-7721, MHCC97L and MHCC97H) were cultivated in DMEM medium supplemented with $10 \%$ fetal calf serum (Sigma Chemical Co., St. Louis, MO, USA). Primary antibodies against PTEN, RECK, PDCD4 and GAPDH were purchased from Santa Cruz Biotechnology (Santa Cruz, CA, USA). All secondary antibodies were obtained from Pierce (Rockford, IL, USA). PTEN, RECK and PDCD4 small interfering RNA (siRNA) and siRNA controls were obtained from Santa Cruz Biotechnology (Santa Cruz, CA, USA). Lipofectamine 2000 was purchased from Invitrogen (Carlsbad, CA, USA). All other chemicals and solutions were purchased from Sigma-Aldrich unless otherwise indicated.

Flow cytometry analysis. To identify and isolate the SP and MP fractions, we used flow cytometry analysis described before (11).

Real-time PCR. Total RNA, including miRNAs, was isolated from prepared liver samples or cells with TRIzol reagent (Invitrogen) according to the manufacturer's instructions. Expression of hsa-miR-21 was analyzed with the miScript system (Qiagen, USA), which consists of the miScript Reverse Transcription kit, miScript Primer assays and miScript SYBR Green PCR kit, according to the protocol provided by the company. Small nuclear RNA U6 was used for normalization. For the analysis of BCRP1, AFP, CK19, PTEN, RECK and PDCD4 expression, cDNA was synthesized using Moloney murine leukaemia virus reverse transcriptase (Epicentre, Paris, France) as described by the manufacturer. The housekeeping gene, glyceraldehyde-3-phosphate dehydrogenase (GAPDH), was used for normalization. Primers used are shown in Table I. Real-time PCR was run on the ABI PRISM 7700 Sequence Detector (Applied Biosystems, USA). All of the reactions were run in triplicate. The ${ }^{\Delta 4} \mathrm{Ct}$ method was used for relative quantification of gene expression to determine miR-21 and BCRP1, AFP, CK19, PTEN, RECK and PDCD4 mRNA expression levels.

Chemo-resistance assay. Sorted SP or MP cells were cultured with the chemotherapeutic agent doxorubicin (DOX, $50 \mathrm{nM}$ ) or methotrexate (MTX, $100 \mathrm{nM})$. After 72-h exposure, cell viability was examined by an MTS-based Cell Titer 96 Aqueous One Solution Cell Proliferation Assay (Promega).
Table I. The primers used in the PCR reaction.

\begin{tabular}{ll}
\hline Gene & \multicolumn{1}{c}{ Primer sequences } \\
\hline BCRP1 & F: 5'-CAACCATTGCATCTTGGCTG-3' \\
& R: 5'-CAAGGCCACGTGATTCTTCC-3' \\
AFP & F:5'-CAGGAGGAAGAAAGGACAAAAAA-3' \\
& R: 5'-ATTCCTAAGGCATAGAAATCCCA-3' \\
CK-19 & F: 5'-ATGGCCGAGCAGAACCGGAA-3' \\
& R: 5'-CCATGAGCCGCTGGTACTCC-3' \\
PTEN & F: 5'-GCGTGCAGATAATGACAAGG-3' \\
& R: 5'-GGATTTGACGGCTCCTCTAC-3' \\
RECK & F: 5'-TGCAAGCAGGCATCTTCAAA-3' \\
& R: 5'-ACCGAGCCCATTTCATTTCTG-3' \\
PDCD4 & F: 5'-AGTGACGCCCTTAGAAGTGG-3' \\
& R: 5'-TCATATCCACCTCCTCCACA-3' \\
GAPDH & F: 5'-CAAGGTCATCCATGACAACTTTG-3' \\
& R: 5'-GTCCACCACCCTGTTGCTGTAG-3'
\end{tabular}

F, forward. R, reverse.

Migration and invasion assays. The cell migration was analyzed with non-Matrigel-coated Transwell cell culture chambers ( $8-\mu \mathrm{m}$ pore size) (Millipore, Billerica, MA, USA). The cell invasion was analyzed with Matrigel-coated Transwell cell culture chambers ( $8-\mu \mathrm{m}$ pore size) (Millipore). Briefly, cells $\left(5 \times 10^{4}\right.$ cells/well) were serum starved for $24 \mathrm{~h}$ and plated in the upper insert of a 24 -well chamber in a serum-free medium. A medium containing $10 \%$ serum or the chemokine CXCL12 (PeproTech) was added to the well. The cells were incubated for $24 \mathrm{~h}$. Cells on the upper side of the filters were mechanically removed by scrubbing with a cotton swab, after which the membrane was fixed with $4 \%$ formaldehyde for $10 \mathrm{~min}$ at room temperature and stained with $0.5 \%$ crystal violet for $10 \mathrm{~min}$. Finally, invasive or migrated cells were counted at magnification x200 from 10 different fields of each filter.

Actin polymerization. Sorted SP or MP cells were resuspended and kept at $37^{\circ} \mathrm{C}$. Human CXCL12 (PeproTech) or PBS were added to cell suspensions and aliquots were taken at the indicated times and immediately fixed in $4 \%$ paraformaldehyde for $10 \mathrm{~min}$. After washing, samples were stained with FITCPhalloidin (Molecular Probes) stain F-actin and analyzed by flow cytometry. The relative F-actin index was determined as the ratio of the F-actin level of SP or MP cells treated with CXCL12 to SP or MP cells treated with PBS.

Cell transfection. MiR-21 mimics, inhibitors (miR-21-AS) and their respective negative controls (NC) were obtained from GenePharma Co. (Shanghai, China). The day before transfection, SP cells were seeded in antibiotic-free medium. Transfections were carried out using Lipofectamine 2000 (Invitrogen) in accordance with the manufacturer's instructions. To monitor transfection efficiency, fluorescein (FAM) siRNA (GenePharma) was used as control. Successfully transfected cells were observed with a fluorescence microscope. According 
A

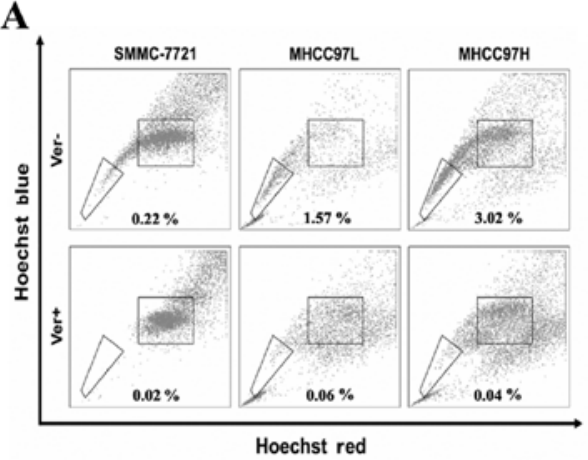

B

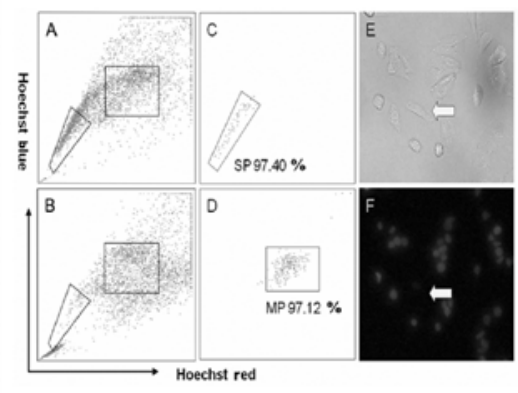

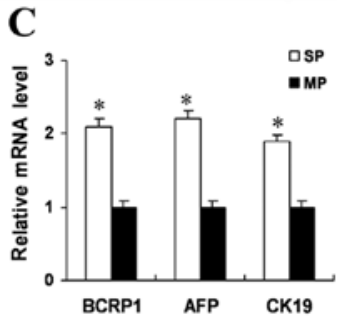

SMMC-7721

D

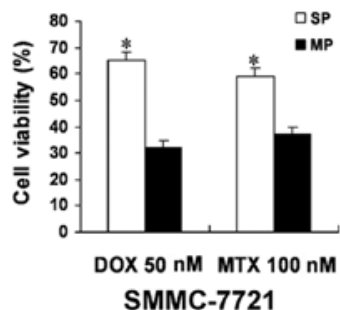

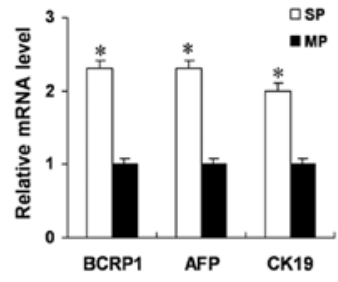

MHCC97L

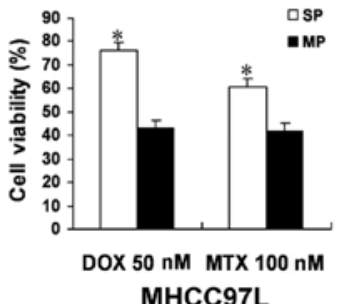

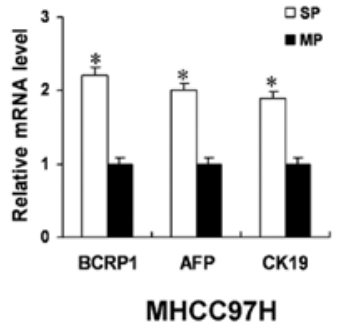

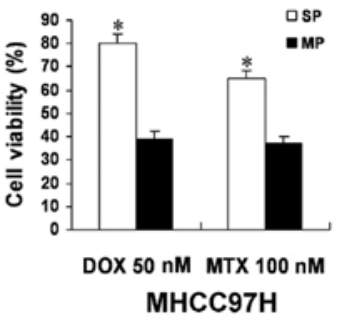

Figure 1. SP cells are an enriched source of stem-like cells in HCC cell lines. (A) Identification of SP and MP cells in the HCC cell lines SMCC-7721, MHCC97L and MHCC97H. (B) The isolated SP cells had high purity and can efflux the DNA-binding dye Hoechst 33342 in MHCC97H. (C) Relative mRNA expression of BCRP1, AFP and CK19 in SP and MP cells was determined using real-time PCR analysis. (D) Chemo-resistance of SP and MP cells to anticancer drugs. Cells were incubated with DOX $(50 \mathrm{nM})$, or MTX $(100 \mathrm{nM})$ for $72 \mathrm{~h}$. Cell viability was then determined using the MTS assay. ${ }^{*} \mathrm{P}<0.05$ compared with MP cells.

to the protocol supplied with the Lipofectamine 2000, SP cells were transfected with either siRNA or control siRNA. siRNAtransfected cells were seeded into 6-well cell culture plates at a density of $1 \times 10^{5}$ cells/well. The cells were allowed to grow for an additional $24 \mathrm{~h}$ and were then harvested for further analysis.

Plasmid construction and luciferase reporter assay. The 3'-UTR of PTEN or RECK or PDCD4 containing the PTEN or RECK or PDCD4-miR-21 response element was cloned into the pIS0 control luciferase vector (Promega). A mutant 3'-UTR of PTEN or RECK or PDCD4 was synthesised by PCR. SP cells were seeded in a 24 -well plate $\left(1 \times 10^{5}\right.$ per well $)$ and transiently transfected with PTEN or RECK or PDCD4-UTR-pIS0/ Mu-PTEN or RECK or PDCD4-UTR-pIS0, Renilla luciferase control vector $(20 \mathrm{ng}$ ) and miR-21/NC. Luciferase activity was measured $48 \mathrm{~h}$ later using a dual luciferase reporter assay system according to the manufacturer's protocol (Promega).

Protein extraction and western blotting. The cells were lysed in lysis buffer [50 mmol/1 Tris (pH 7.5), $100 \mathrm{mmol} / \mathrm{l} \mathrm{NaCl}$, $1 \mathrm{mmol} / \mathrm{l}$ EDTA, $0.5 \%$ NP40, 0.5\% Triton X-100, $2.5 \mathrm{mmol} / 1$ sodium orthovanadate, $10 \mu \mathrm{l} / \mathrm{ml}$ protease inhibitor cocktail and $1 \mathrm{mmol} / 1 \mathrm{PMSF}$ ] by incubating for $20 \mathrm{~min}$ at $4^{\circ} \mathrm{C}$. The protein concentration was determined using the Bio-Rad assay system (Bio-Rad, Hercules, CA, USA). Total proteins were fractionated using SDS-PAGE and transferred onto nitrocellulose membranes. The membranes were blocked with $5 \%$ non-fat dried milk or bovine serum albumin in 1X TBS buffer containing $0.1 \%$ Tween-20 and then incubated with the appropriate primary antibodies. Horseradish peroxidase-conjugated anti-rabbit or anti-mouse IgG was used as the secondary antibody and the protein bands were detected using the enhanced chemiluminescence detection system (Amersham Pharmacia Biotech). Quantification of the western blots was performed using laser densitometry and relative protein expression was then normalized to GAPDH levels.

Statistical analysis. Each experiment was repeated at least three times. All data are summarized and presented as means \pm SDs. The differences among means were statistically analyzed using a t-test. All statistical analyses were performed using SPSS 13.0 software (Chicago, IL, USA). $\mathrm{P}<0.05$ was considered as statistically significant.

\section{Results}

SP cells are an enriched source of stem-like cells in HCC cell lines. Using flow cytometry, we were able to identify and successfully isolate populations of SP and MP cells from the three human HCC cell lines (Fig. 1A). The SP 
$\mathbf{A}$

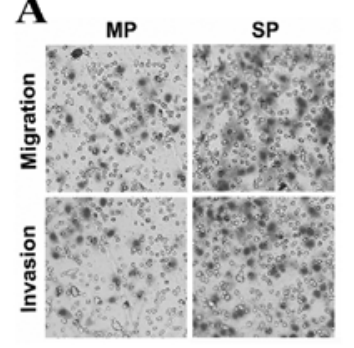

SMMC-7721

B

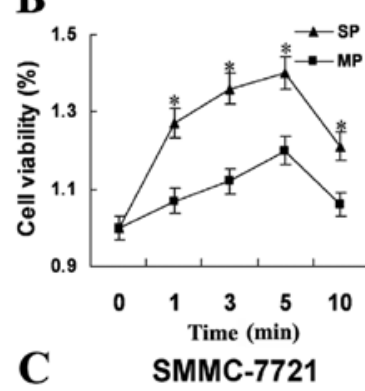

C

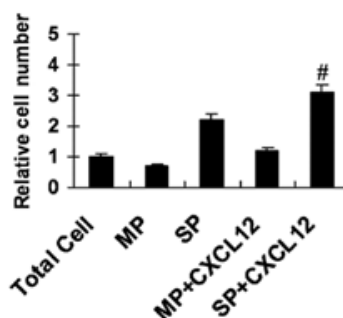

SMMC-7721

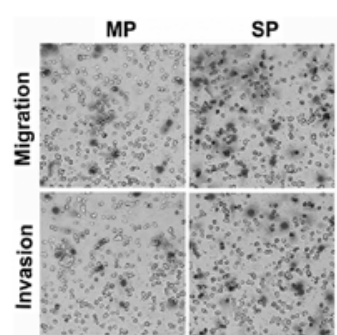

MHCC97L

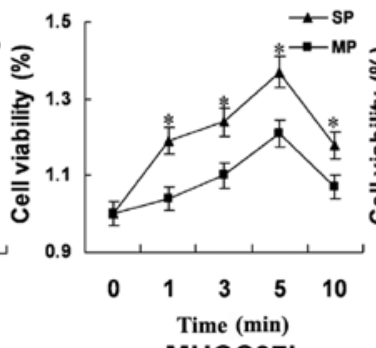

MHCC97L

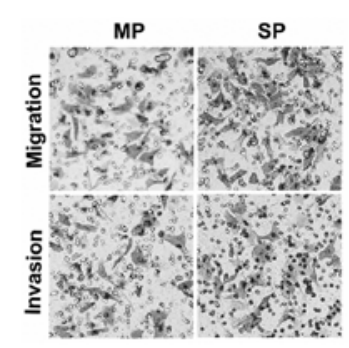

MHCC97H

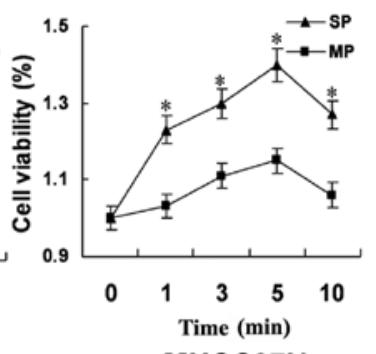

$\mathrm{MHCC} 97 \mathrm{H}$
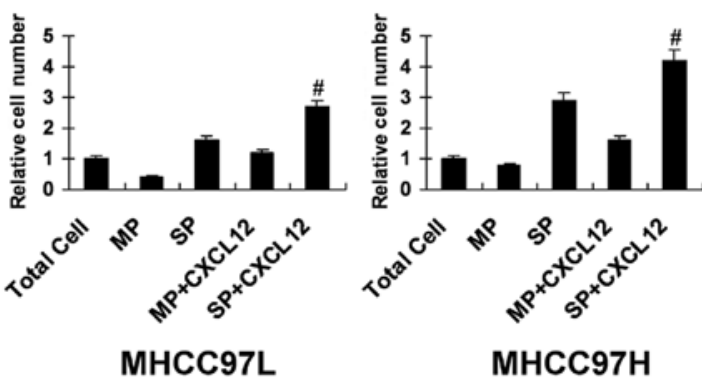

Figure 2. SP cells show more migration and invasion capability than MP cells. (A) The invasion and migration of SP and MP cells were measured by Transwell cell culture chambers. (B) The effect of CXCL12 on actin polarization. F-actin levels were examined at the indicated time-points after CXCL12 stimulation. Phalloidin staining was then analyzed by flow cytometry. The relative F-actin index was determined as the ratio of the F-actin levels of cells treated with CXCL12 to cells treated with PBS. (C) The effect of CXCL12 on cell migration. A transwell system was used to examine cell migration. The relative migration index was determined as the ratio of the number of migrated cells in each group to that of total cell group. ${ }^{*} \mathrm{P}<0.05$ compared with $\mathrm{MP}$ cells. ${ }^{\#} \mathrm{P}<0.05$ compared with MP cells + CXCL12.

gate was defined as the region where cells were absent in the presence of verapamil, an agent which blocks the efflux of Hoechst 33342. The three cell lines, SMCC-7721, MHCC97L and MHCC97H, contained 0.19 $\pm 0.02,1.34 \pm 0.06$ and $2.95 \pm 0.24 \%$ SP cells, respectively. Since MHCC $97 \mathrm{H}$ contained the highest percentage of SP cells further experiments were performed using these cells. The isolated SP cells had high purity and can efflux the DNA-binding dye Hoechst 33342 in MHCC97H (Fig. 1B). It showed similar results in MHCC97L and SMMC-7721 cells (data not shown). Expression of the hepatocyte-specific marker AFP and the cholangiocyte-specific marker CK19 has been associated with subpopulations showing bipotential stem/progenitor properties in HCC. BCRP1 has the capacity to efflux a broad range of cytotoxic substances and is characteristic of the SP phenotype. These genes were expressed in both SP and MP cells but upregulated in SP cells relative to MP cells (Fig. 1C). The chemo-resistance ability of SP cells has been reported to depend mainly on ABC transporters (17). To determine whether SP cells are able to resist ABC transporter-independent anticancer drugs more than MP cells we tested DOX and MTX which are used for the treatment of HCC (Fig. 1D). SP cells were more resistant than MP cells, especially to DOX. Cells were then stained with Hoechst 33342 and reanalyzed. A higher percentage of SP cells were seen after $72 \mathrm{~h}$ of DOX or MTX treatment. This suggested that SP cells might be more resistant to anticancer drugs than MP cells. We also evaluated other data such as repopulation capability and cell cycle analysis described before (11). It showed similar results to our previous study (11). From these data, we can propose SP cells exhibit stem-like characteristics.

SP cells show more migration and invasion capabilities than MP cells. CSC in a tumor is proposed to mediate invasion and metastasis and SP cells from tumor cells possess the properties ascribed to CSC. Fig. 2A shows SP cells with higher levels of penetration through Transwell cell culture chambers versus the MP cells. Cell polarization requires actin polymerization (18). As shown in Fig. 2B, CXCL12 treatment increased the extent of actin polymerization in SP cells more than MP cells at the studied time-points. Following treatment with CXCL12, the relative migrating index of SP cells was higher than MP cells (Fig. 2C), suggested SP cells show more potentiality of migration and invasion than MP cells. 
$\mathbf{A}$

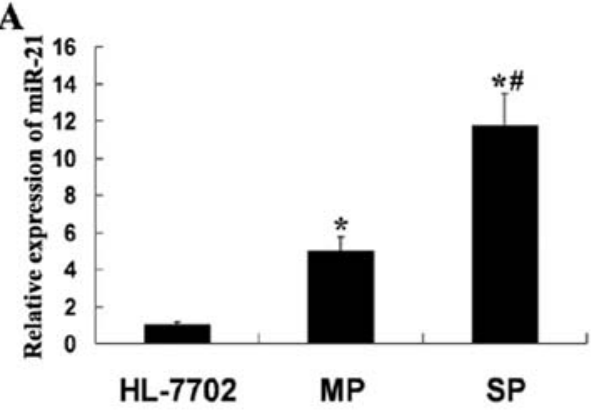

B

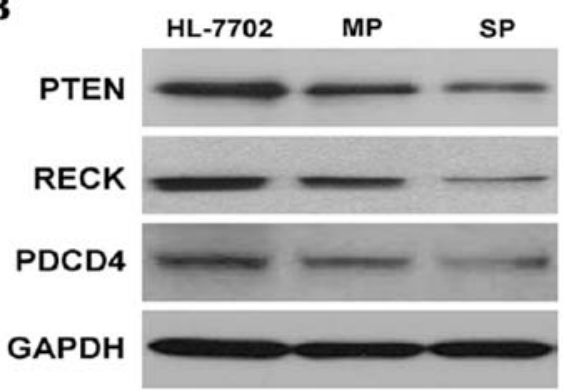

Figure 3. The expression of miR-21, PTEN, RECK and PDCD4. (A) The expression of miR-21 was measured by RT-PCR. (B) The protein expressions of PTEN, RECK and PDCD4 were measured by western blotting. "P $<0.05$ compared with HL-7702 cells. ${ }^{*} \mathrm{P}<0.05$ compared with MP cells.

A

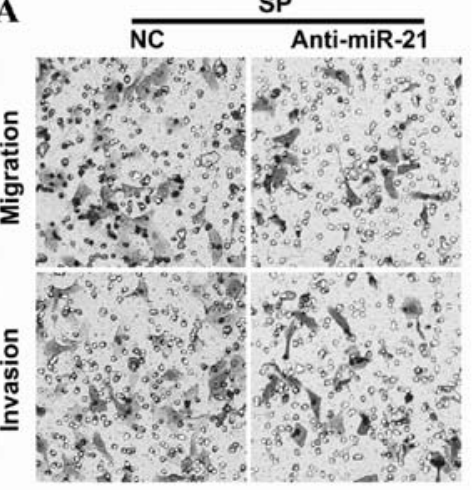

C

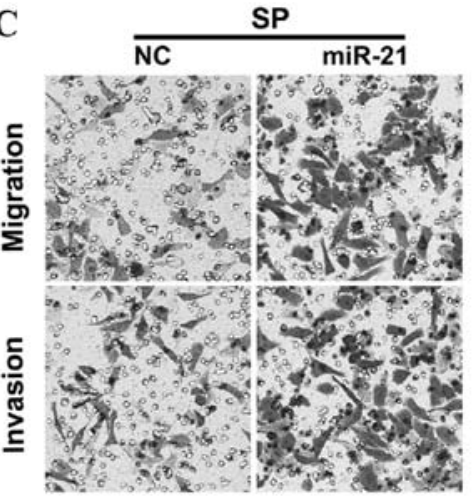

B

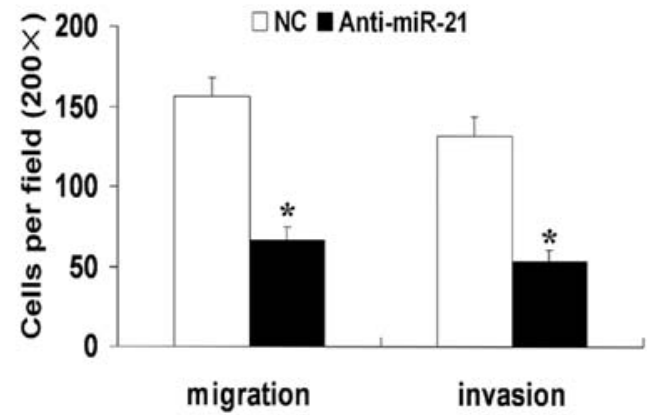

D

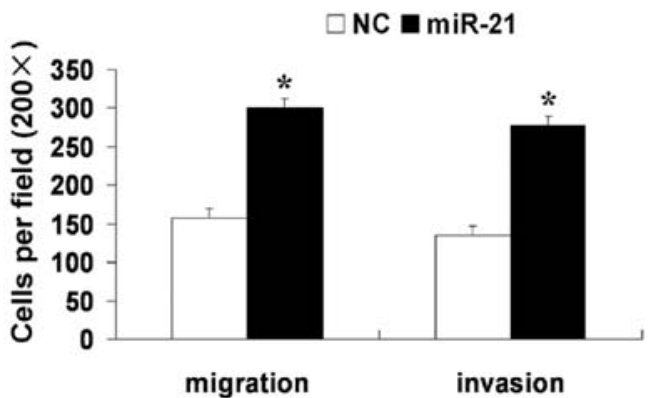

Figure 4. MiR-21 promotes SP cells migration and invasion. (A and B) Transwell migration assay and Matrigel invasion assay of SP cells transfected with miR10b-AS or NC. (C and D) Transwell migration assay and Matrigel invasion assay of SP cells transfected with miR-10b mimics or NC. "P<0.05 compared with NC.

MiR-21 promotes $S P$ cell migration and invasion by targeting $P T E N$, RECK and PDCD4. Increasing number of reports implicate miR-21 overexpression in carcinogenesis and metastasis. To assess the relevance of miR-21 in SP cells, we first determined its expression level comparing MP cells and liver non-tumor HL-7702 cells. Interestingly, compared with HL-7702 cells, miR-21 expression was found to be upregulated in SP and MP cells. These results indicated miR-21 may be important in HCC. Furthermore, the higher expression of miR-21 was observed in SP cells than MP cells (Fig. 3A). As a previous study described, miR-21 can regulate HCC cells migration and invasion by targeting PTEN, RECK and PDCD4 (13). We also determined the protein level of PTEN, RECK and PDCD4 in HL-7702, MP and SP cells. The lower expression of PTEN, RECK and PDCD4, respectively, was observed in SP cells than MP and HL-7702 cells (Fig. 3B). There was an inverse correlation between miR-21 and its target gene. These results also indicated as in the cancer cells, miR-21 may play a similar role in CSC.

An important component of the invasive profile of a cancer cell is its ability to be motile. High levels of miR-21 have been associated with cell motility and metastasis of different cancers. Having this background in mind, we first performed in vitro loss-of-function analyses by silencing miR-21 with miR-21-AS in SP cells. Silencing of miR-21 led to a more than $60 \%$ reduction in the migration and invasion of these cells (Fig. 4A and B). Next, synthetic miR-21 mimics or NC were transfected into SP cells and overexpression of miR-21 caused a 2 -fold increase in cell migration and invasion (Fig. 4C and D). These results confirmed the involvement 

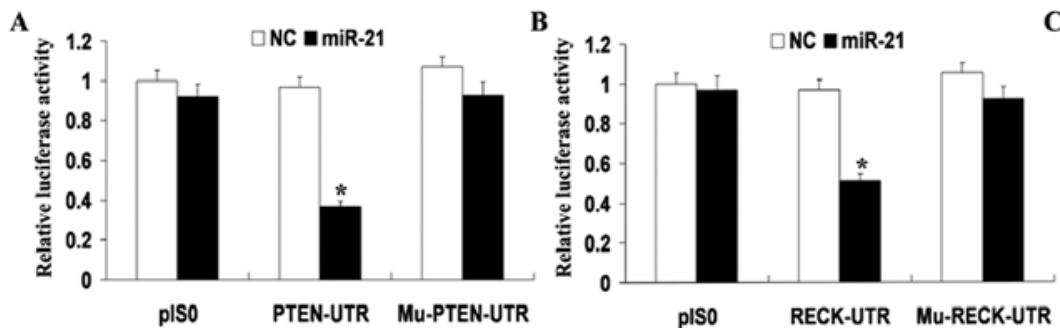
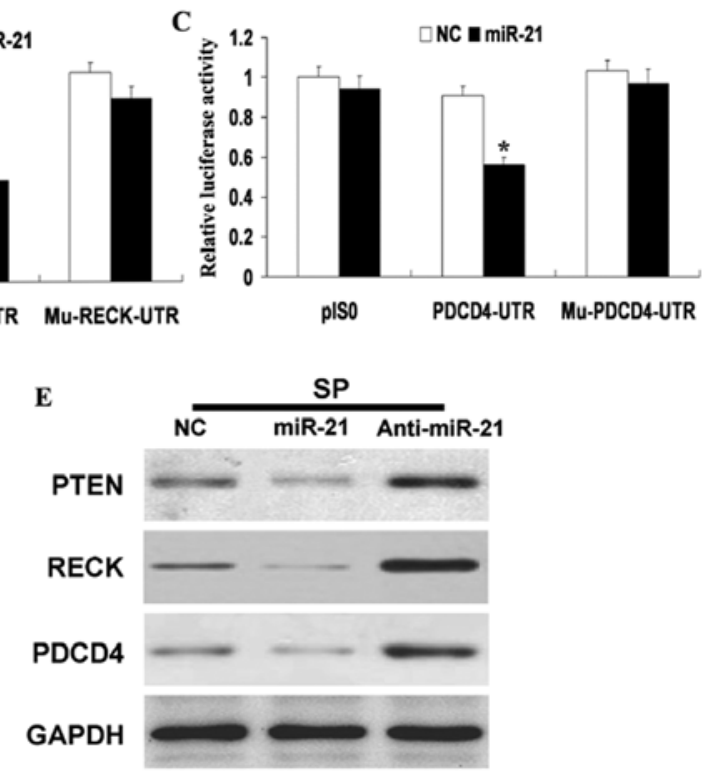

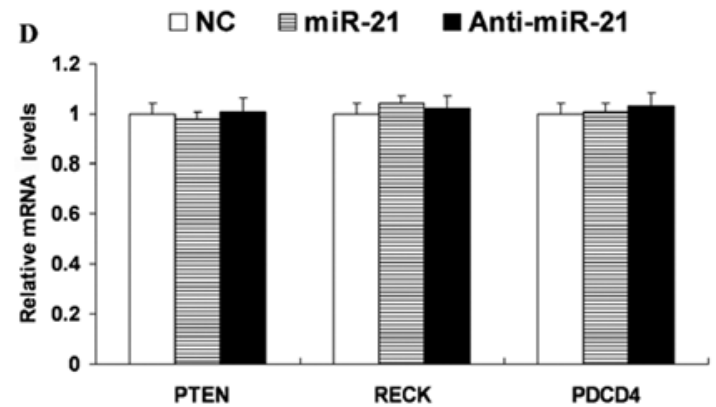

Figure 5. PTEN, RECK and PDCD4 are targets of miR-21. (A-C) Luciferase activity of SP cells co-transfected with PTEN, RECK or PDCD4-UTR-pIS0/ Mu-PTEN or RECK or PDCD4-UTR-pIS0 and miR-21 mimics or NC. The pIS0 control vector was used as a control ("P<0.05). (D) SP cells were transfected with miR-21 mimics or miR-21-AS or NC. The mRNA levels of PTEN, RECK and PDCD4 protein were analyzed by RT-PCR. (E) SP cells were transfected with miR-21 mimics or miR-21-AS or NC. The protein levels of PTEN, RECK and PDCD4 protein were analyzed by western blotting.

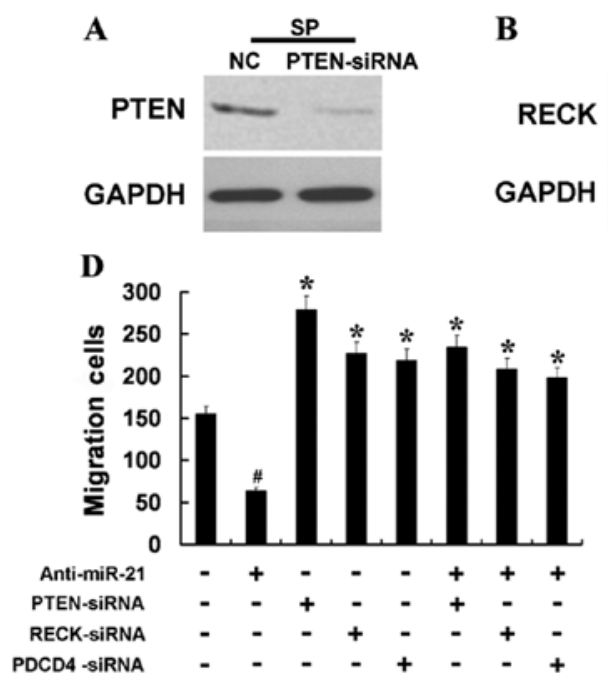

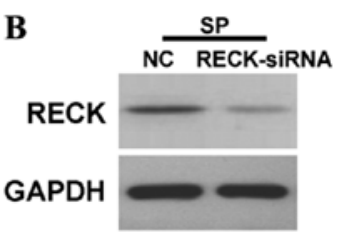
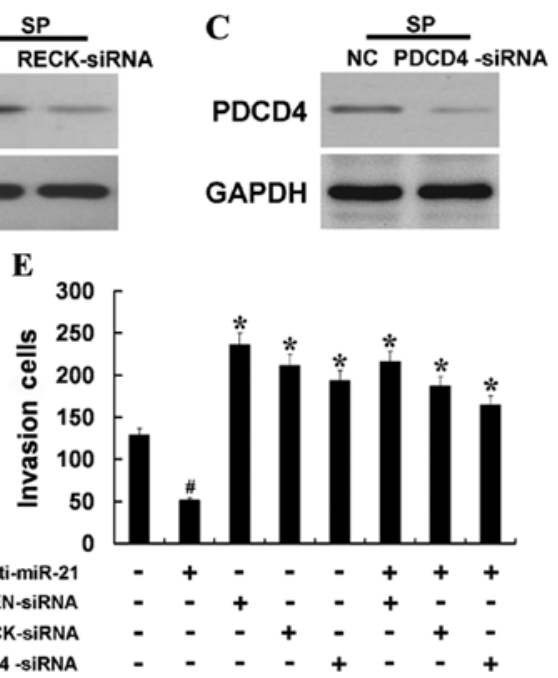

Figure 6. The effect of miR-21 on migration and invasion of SP cells was partly mediated through regulation of PTEN, RECK or PDCD4 expression. (A-C) The levels of PTEN, RECK or PDCD4 protein, respectively, in SP cells transfected with siRNA were analyzed by western blotting. (D and E) The effect of miR-21-AS was partially attenuated by silencing of PTEN or RECK or PDCD4 mRNA. ${ }^{*} \mathrm{P}<0.05$ compared with non-treated SP cells. ${ }^{~} \mathrm{P}<0.05$ compared with SP cells only transfected with miR-21-AS.

of miR-21 in the regulation of SP cell motility and suggested a biological role for miR-21 in controlling SP cell ability for migration and invasion.

MiR-21 simultaneously regulates multiple programs that enhance tumor invasiveness by targeting PTEN, PDCD4 and RECK in HCC cells (13). As shown in Fig. 5A-C, miR-21 overexpression remarkably repressed the expression of luciferase containing a wild-type miR-21 binding site (PTEN or RECK or PDCD4-UTR) compared with NC. This suppression was restored by mutations in the seed complementary sites of the 3'-UTR of PTEN or RECK or PDCD4 (Fig. 5A-C). To determine whether miR-21 expression represses endogenous PTEN or RECK or PDCD4 expression through translational repression, synthetic miR-21 mimics and NC were transfected into SP cells. RT-PCR and western blot analyses revealed that miR-21 overexpression did not cause degradation of PTEN or RECK or PDCD4 mRNA (Fig. 5D) but did drastically inhibit its protein expression (Fig. 5E). Consistent with these results, silencing miR-21 with miR-10b-AS, respectively, increased the level of PTEN, RECK or PDCD4 protein (Fig. 5E) but did not alter PTEN, RECK or PDCD4 mRNA levels (Fig. 5D). We examined whether a reduction in the expression of PTEN or RECK or PDCD4 might mediate the induction of cell 
migration and invasion observed following miR-21 overexpression. Silencing of PTEN, RECK or PDCD4 with siRNA in SP cells led to increased cell migration and invasion (Fig. 6D and E), demonstrating a negative role for PTEN, RECK or PDCD4 in the migration and invasion of SP cells. The knockdown efficiency of PTEN, RECK or PDCD4 was verified by western blot analysis (Fig. 6A-C). Further, we co-transfected SP cells with siRNA for PTEN, RECK and PDCD4 mRNA, respectively, and miR-21-AS and found that the effect of miR21-AS was partially attenuated by silencing of PTEN, RECK or PDCD4 mRNA (Fig. 6D and E). These results indicated that the prometastatic effect of miR-21 is partly mediated through regulation of PTEN, RECK or PDCD4 expression.

\section{Discussion}

Hepatocellular carcinoma (HCC) is the most common malignancy of the liver and surgical resection remains the major treatment. However, long-term results after resection of HCC are still unsatisfactory. The main cause for the poor prognosis is the high recurrence rate and a risk of vascular invasion, and metastasis even after curative resection of HCC. Therefore, further improvement of long-term survival after hepatic resection may depend on prevention and treatment of the recurrent tumor, which has attracted attention of researchers in recent years. Although studies have focused on HCC invasion and metastasis, the underlying mechanisms are still not fully understood (19).

There is increasing evidence indicating that the maintenance and spreading of a variety of tumors is sustained by a small subset of cancer cells, termed CSC. These cells possess the ability for self-renewal, unlimited proliferation potential and capacity to generate differentiated cells which constitute the major tumor population (20). Currently, human CSC have been identified in a variety of tumor types $(5,21-23)$. It has been reported that CSCs are resistant to chemotherapy and targeted therapy, which resulted in cancer relapse and metastasis. An important criterion is that tumors with high percentages of cancer stem cells will be more aggressive (17). On the other hand, in the analysis of hematopoietic stem cells, a sub-population that effluxes the DNA-binding dye Hoechst 33342 out of the cell membrane through an ATP-binding cassette (ABC) transporter was recognised as a stem cell population (7). This cell population expressing the ABC transporter was defined as side population (SP) cells, which were distinguished from cells of the other population (main population; MP). Moreover, recent work has led to the detection of the SP cells in a variety of tumor types $(8,10,24)$. SP cells from tumor cells possess the properties ascribed to cancer stem cells and have been proposed to play a critical role in metastasis progression (20). Our present results showed that SP cells had more migration and invasion capabilities than MP cells, therefore, this may be one of the potential mechanisms of $\mathrm{HCC}$ cell invasion and metastasis.

MiRNAs have a broad impact on gene expression through translational repression or post-transcriptional suppression (25). MiRNAs play important roles in multiple biological processes such as development, differentiation and cellular stress response. Previous studies have linked deregulation of miRNAs to various diseases including cancer $(26,27)$. A single miRNA can potentially bind to hundreds of mRNA targets, thereby having an important role in various biological processes (28). Furthermore, it has been demonstrated that miRNAs can induce tumorigenesis, for example, by downregulating tumor suppressor genes. Among such oncogenic miRNAs, miR-21 has been shown to be overexpressed in a variety of malignancies (29-31). Therefore, miR-21 has been recognized as an oncomir. Elevated miR-21 expression has been causally linked to cell metastasis $(32,33)$. In our data, expression of miR-21 was more significantly increased in SP cells compared to MP cells and changes of miR-21 can regulate migration and invasion of SP cells. The link between miR-21 and SP cell metastasis suggests the presence of metastatic pathways and raised the question regarding the mechanisms of how miR-21 may impact the metastatic potential of SP cells.

The metastatic process is complex and often associated with alterations in the different metastasis-related protein of the tumor cells. PTEN is a phosphoinositide phosphatase which was originally identified as a multifunctional tumor suppressor frequently lost in various human cancers $(34,35)$. PTEN expression is associated with tumor invasion and tumor-node-metastasis (TNM) stage $(36,37)$. As a tumor suppressor in multiple cancers including HCC, PTEN can affects the Akt and ERK signaling pathways (38). These pathways are linked to cell survival, proliferation, differentiation, cell migration and invasion. Previous studies showed the modulation of expression of PTEN in HCC can impact on the activity of critical downstream mediators of tumor progression and metastases (14). RECK, a membraneanchored glycoprotein, is able to suppress tumor invasion and metastasis and is known to inhibit both catalytic and processing steps of pro-MMP-2 activity and associated with the suppression of MMP-2, MMP-9 and MMP-14 secretion $(39,40)$. In tumors, RECK is absent or diminished and MMPs are highly active, facilitating tumor promotion and progression. The expression of RECK is often decreased during cancer progression $(41,42)$ and is a molecular marker for cancer prognosis and controller of cellular metastatic capacity (40). RECK expression levels are predictive in determining prognoses in a number of common cancers and low levels of RECK are often associated with increased invasiveness and poor prognosis $(43,44)$. Programmed cell death 4 (PDCD4) is a tumor suppressor gene that inhibits metastasis in human cancer cells (45). PDCD4 is a downstream component of the Akt pathway and PDCD4 has been shown to inhibit neoplastic transformation, tumor development and malignant progression $(46,47)$. Studies investigating cellular functions of PDCD4 demonstrated that it suppresses the expression and/or activity of the invasion-related proteins such as AKT (48), MAP4K1 (49) and increases the release of metastasis suppressor proteins such as E-cadherin (50) and TIMP2 (51). In hepatocellular carcinoma cells, PDCD4 has recently been shown to correlate inversely with metastatic capacity (52). Thus, change of these proteins can be involved in migrating and invading of tumor cells. Our studies showed that the protein of PTEN, RECK or PCCD4 decreased in SP cells. The results indicated these proteins may participate in migration and invasion of SP cells. A few targets of miR-21 have been experimentally validated, including some metas- 
tasis-related protein such as PTEN (14), PDCD4 (15), RECK (16). Recent studies had showed that miR-21 simultaneously regulates multiple programs that enhance tumor invasiveness by targeting PTEN, PDCD4 and RECK in HCC cells (13). We hypothesized that miR-21 may play an important role in regulating migration and invasion of cancer stem-like cells by targeting PTEN, PDCD4 and RECK.

Although PTEN, PDCD4 and RECK are direct targets of miR-21 and their expression is inhibited by miR-21 in HCC cells, we still question whether miR-21 could regulate these proteins in cancer stem-like cells. One of the best ways to understand miRNA function is via the elucidation of functional targets, which usually involves analysis of changes in target proteins following either a gain- or loss-of function of the specific miRNA. In our present study, we also found that miR-21 overexpression drastically inhibits PTEN, RECK or PDCD4 protein expression and silencing miR-21 increased the level of PTEN, RECK or PDCD4 protein. On the other hand, we also observed that SP cell migration and invasiveness weakened by anti-miR-21 was 'rescued' by knockdown of PTEN, PDCD4 or RECK. Taken together the biological effects of miR-21 on SP cell invasion are probably due to the simultaneous repression of migration suppressive proteins such as PTEN, RECK or PDCD4.

In conclusion, the results of this study revealed the aberrant expression of miR-21 in SP cells and showed that miR-21 regulates the expression of multiple target proteins that are associated with tumor dissemination, many of which are implicated in SP cell biology. Importantly, repression of miR-21 inhibited SP cell migration and invasion in vitro, possibly due to downregulation of the tumor suppressor PTEN, RECK or PDCD4. These compelling data provide preliminary evidence that miR-21 is a pro-metastatic miRNA in SP cells and raise the possibility that therapy of HCC may be improved by pharmaceutical strategies directed towards miR-21.

\section{Acknowledgements}

We are grateful to Fu-Gin Zhang for technical help. This study was supported by grants from the National Natural Science Foundation of China (grants no. 81101619/H1607).

\section{References}

1. Lau WY and Lai EC: Hepatocellular carcinoma: current management and recent advances. Hepatobiliary Pancreat Dis Int 7: 237-257, 2008.

2. Visvader JE and Lindeman GJ: Cancer stem cells in solid tumours: accumulating evidence and unresolved questions. Nat Rev Cancer 8: 755-768, 2008.

3. Simeone DM: Pancreatic cancer stem cells: implications for the treatment of pancreatic cancer. Clin Cancer Res 14: 5646-5648, 2008

4. Ho MM, Ng AV, Lam S and Hung JY: Side population in human lung cancer cell lines and tumors is enriched with stem-like cancer cells. Cancer Res 67: 4827-4833, 2007.

5. Prince ME, Sivanandan R, Kaczorowski A, et al: Identification of a subpopulation of cells with cancer stem cell properties in head and neck squamous cell carcinoma. Proc Natl Acad Sci USA 104: 973-978, 2007.

6. Wang J, Guo LP, Chen LZ, Zeng YX and Lu SH: Identification of cancer stem cell-like side population cells in human nasopharyngeal carcinoma cell line. Cancer Res 67: 3716-3724, 2007.
7. Goodell MA, Brose K, Paradis G, Conner AS and Mulligan RC: Isolation and functional properties of murine hematopoietic stem cells that are replicating in vivo. J Exp Med 183: 1797-1806, 1996.

8. Kondo T, Setoguchi T and Taga T: Persistence of a small subpopulation of cancer stem-like cells in the C6 glioma cell line. Proc Natl Acad Sci USA 101: 781-786, 2004.

9. Kruger M, Schwarz A and Blumich B: Investigations of silicone breast implants with the NMR-MOUSE. Magn Reson Imaging 25: 215-218, 2007.

10. Mitsutake N, Iwao A, Nagai K, et al: Characterization of side population in thyroid cancer cell lines: cancer stem-like cells are enriched partly but not exclusively. Endocrinology 148: 1797-1803, 2007.

11. Zhang N, Li R, Tao KS, et al: Characterization of a stem-like population in hepatocellular carcinoma MHCC97 cells. Oncol Rep 23: 827-831, 2010.

12. Ambros V: MicroRNA pathways in flies and worms: growth, death, fat, stress and timing. Cell 113: 673-676, 2003.

13. Liu C, Yu J, Yu S, et al: MicroRNA-21 acts as an oncomir through multiple targets in human hepatocellular carcinoma. J Hepatol 53: 98-107, 2010

14. Meng F, Henson R, Wehbe-Janek H, Ghoshal K, Jacob ST and Patel T: MicroRNA-21 regulates expression of the PTEN tumor suppressor gene in human hepatocellular cancer. Gastroenterology 133: 647-658, 2007.

15. Asangani IA, Rasheed SA, Nikolova DA, et al: MicroRNA-21 (miR-21) post-transcriptionally downregulates tumor suppressor Pdcd 4 and stimulates invasion, intravasation and metastasis in colorectal cancer. Oncogene 27: 2128-2136, 2008.

16. Gabriely G, Wurdinger T, Kesari S, et al: MicroRNA 21 promotes glioma invasion by targeting matrix metalloproteinase regulators. Mol Cell Biol 28: 5369-5380, 2008.

17. Dean M, Fojo T and Bates S: Tumour stem cells and drug resistance. Nat Rev Cancer 5: 275-284, 2005.

18. Devreotes P and Janetopoulos C: Eukaryotic chemotaxis: distinctions between directional sensing and polarization. J Biol Chem 278: 20445-20448, 2003.

19. Tang DJ, Dong SS, Ma NF, et al: Overexpression of eukaryotic initiation factor 5A2 enhances cell motility and promotes tumor metastasis in hepatocellular carcinoma. Hepatology 51: 1255-1263, 2010.

20. Reya T, Morrison SJ, Clarke MF and Weissman IL: Stem cells, cancer and cancer stem cells. Nature 414: 105-111, 2001.

21. Al-Hajj M, Wicha MS, Benito-Hernandez A, Morrison SJ and Clarke MF: Prospective identification of tumorigenic breast cancer cells. Proc Natl Acad Sci USA 100: 3983-3988, 2003.

22. Matsui W, Huff CA, Wang Q, et al: Characterization of clonogenic multiple myeloma cells. Blood 103: 2332-2336, 2004.

23. Singh SK, Hawkins C, Clarke ID, et al: Identification of human brain tumour initiating cells. Nature 432: 396-401, 2004.

24. Szotek PP, Pieretti-Vanmarcke R, Masiakos PT, et al: Ovarian cancer side population defines cells with stem cell-like characteristics and Mullerian Inhibiting Substance responsiveness. Proc Natl Acad Sci USA 103: 11154-11159, 2006.

25. Ambros V: The functions of animal microRNAs. Nature 431: $350-355,2004$

26. Lu J, Getz G, Miska EA, et al: MicroRNA expression profiles classify human cancers. Nature 435: 834-838, 2005.

27. Iorio MV, Visone R, Di Leva G, et al: MicroRNA signatures in human ovarian cancer. Cancer Res 67: 8699-8707, 2007.

28. Bartel DP: MicroRNAs: genomics, biogenesis, mechanism and function. Cell 116: 281-297, 2004.

29. Bloomston M, Frankel WL, Petrocca F, et al: MicroRNA expression patterns to differentiate pancreatic adenocarcinoma from normal pancreas and chronic pancreatitis. JAMA 297: 1901-1908, 2007.

30. Feber A, Xi L, Luketich JD, et al: MicroRNA expression profiles of esophageal cancer. J Thorac Cardiovasc Surg 135: 255-260, 2008.

31. Yanaihara N, Caplen N, Bowman E, et al: Unique microRNA molecular profiles in lung cancer diagnosis and prognosis. Cancer Cell 9: 189-198, 2006.

32. Krichevsky AM and Gabriely G: miR-21: a small multi-faceted RNA. J Cell Mol Med 13: 39-53, 2009.

33. Grunder E, D'Ambrosio R, Fiaschetti G, et al: MicroRNA-21 suppression impedes medulloblastoma cell migration. Eur J Cancer 47: 2479-2490, 2011.

34. Li J, Yen C, Liaw D, et al: PTEN, a putative protein tyrosine phosphatase gene mutated in human brain, breast and prostate cancer. Science 275: 1943-1947, 1997. 
35. Salmena L, Carracedo A and Pandolfi PP: Tenets of PTEN tumor suppression. Cell 133: 403-414, 2008.

36. Bedolla R, Prihoda TJ, Kreisberg JI, et al: Determining risk of biochemical recurrence in prostate cancer by immunohistochemical detection of PTEN expression and Akt activation. Clin Cancer Res 13: 3860-3867, 2007.

37. Yoshimoto M, Cunha IW, Coudry RA, et al: FISH analysis of 107 prostate cancers shows that PTEN genomic deletion is associated with poor clinical outcome. Br J Cancer 97: 678-685, 2007.

38. Yu J, Zhang SS, Saito K, et al: PTEN regulation by Akt-EGR1ARF-PTEN axis. EMBO J 28: 21-33, 2009.

39. Noda M, Oh J, Takahashi R, Kondo S, Kitayama H and Takahashi C: RECK: a novel suppressor of malignancy linking oncogenic signaling to extracellular matrix remodeling. Cancer Metastasis Rev 22: 167-175, 2003.

40. Takahashi C, Sheng Z, Horan TP, et al: Regulation of matrix metalloproteinase-9 and inhibition of tumor invasion by the membrane-anchored glycoprotein RECK. Proc Natl Acad Sci USA 95: 13221-13226, 1998.

41. Clark JC, Thomas DM, Choong PF and Dass CR: RECK - a newly discovered inhibitor of metastasis with prognostic significance in multiple forms of cancer. Cancer Metastasis Rev 26 675-683, 2007.

42. Noda M and Takahashi C: Recklessness as a hallmark of aggressive cancer. Cancer Sci 98: 1659-1665, 2007.

43. Kotzsch M, Farthmann J, Meye A, et al: Prognostic relevance of uPAR-del4/5 and TIMP-3 mRNA expression levels in breast cancer. Eur J Cancer 41: 2760-2768, 2005.

44. Takenaka K, Ishikawa S, Kawano Y, et al: Expression of a novel matrix metalloproteinase regulator, RECK and its clinical significance in resected non-small cell lung cancer. Eur J Cancer 40: 1617-1623, 2004.
45. Allgayer H: Pdcd4, a colon cancer prognostic that is regulated by a microRNA. Crit Rev Oncol Hematol 73: 185-191, 2010.

46. Cmarik JL, Min H, Hegamyer G, et al: Differentially expressed protein Pdcd4 inhibits tumor promoter-induced neoplastic transformation. Proc Natl Acad Sci USA 96: 14037-14042, 1999.

47. Jansen AP, Camalier CE and Colburn NH: Epidermal expression of the translation inhibitor programmed cell death 4 suppresses tumorigenesis. Cancer Res 65: 6034-6041, 2005.

48. Lankat-Buttgereit B and Goke R: The tumour suppressor Pdcd4: recent advances in the elucidation of function and regulation. Biol Cell 101: 309-317, 2009.

49. Yang HS, Matthews CP, Clair T, et al: Tumorigenesis suppressor Pdcd4 down-regulates mitogen-activated protein kinase kinase kinase kinase 1 expression to suppress colon carcinoma cell invasion. Mol Cell Biol 26: 1297-1306, 2006.

50. Wang Q, Sun Z and Yang HS: Downregulation of tumor suppressor Pdcd4 promotes invasion and activates both betacatenin/Tcf and AP-1-dependent transcription in colon carcinoma cells. Oncogene 27: 1527-1535, 2008.

51. Nieves-Alicea R, Colburn NH, Simeone AM and Tari AM: Programmed cell death 4 inhibits breast cancer cell invasion by increasing tissue inhibitor of metalloproteinase-2 expression. Breast Cancer Res Treat 114: 203-209, 2009.

52. Zhang S, Li J, Jiang Y, Xu Y and Qin C: Programmed cell death 4 (PDCD4) suppresses metastastic potential of human hepatocellular carcinoma cells. J Exp Clin Cancer Res 28: 71, 2009. 